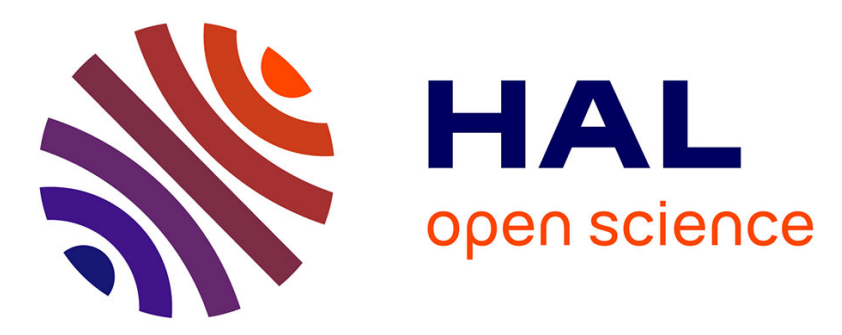

\title{
Uniting two Control Lyapunov Functions for affine Systems
}

Vincent Andrieu, Christophe Prieur

\section{To cite this version:}

Vincent Andrieu, Christophe Prieur. Uniting two Control Lyapunov Functions for affine Systems. Decision and Control, 2008. CDC 2008. 47th IEEE Conference on, Dec 2008, Cancun, Mexico. pp.622 - 627, 10.1109/CDC.2008.4738835 . hal-00457825

\section{HAL Id: hal-00457825 \\ https://hal.science/hal-00457825}

Submitted on 18 Feb 2010

HAL is a multi-disciplinary open access archive for the deposit and dissemination of scientific research documents, whether they are published or not. The documents may come from teaching and research institutions in France or abroad, or from public or private research centers.
L'archive ouverte pluridisciplinaire HAL, est destinée au dépôt et à la diffusion de documents scientifiques de niveau recherche, publiés ou non, émanant des établissements d'enseignement et de recherche français ou étrangers, des laboratoires publics ou privés. 


\title{
Uniting two Control Lyapunov Functions for affine systems
}

\author{
Vincent Andrieu and Christophe Prieur
}

\begin{abstract}
We consider the problem of piecing together two Control Lyapunov Functions (CLFs). The first CLF characterizes a local controllability property toward the origin, whereas the second CLF satisfies a global controllability property with respect to a compact set. We give a sufficient condition to express explicitly a solution to this uniting problem. This sufficient condition is shown to be always satisfied for a simple chain of integrator. In a second part, we show how this uniting CLF problem can be useful to solve the problem of piecing together two stabilizing control laws.
\end{abstract}

\section{INTRODUCTION}

Smooth Control Lyapunov Functions (CLFs) are instrumental in many feedback control designs. See for instance the appropriate sections in the textbooks [6], [8], [10], [11]. The theory of smooth CLF can be traced back to Artstein who introduced this Lyapunov characterization of controllability in [2]. See also [20] for nonsmooth CLFs, and [3] for application of the latter. A very useful characteristic of CLFs is the existence of 'universal formulas' for stabilization of nonlinear control systems that are affine in their controls (see [5], [14], [21]). Also from the converse Lyapunov theory, if the origin of a nonlinear system is robustly asymptotically stabilizable, then there exists a smooth CLF (see [4] or [13]).

Numerous tools for the design of global CLF are now available (for instance by backstepping [6], [11], [17] or by forwarding [9], [16], [19]). On another hand, via linearization (or other local approaches), one may design local CLF yielding locally stabilizing controllers. This leads us to the idea of uniting a local CLF with a global CLF, i.e. given (1) a local CLF $V_{0}$ (e.g. a quadratic CLF yielding to local highperformance stabilizing feedbacks) and (2) a global CLF $V_{\infty}$, we are looking for a third CLF $V$ which is equal (up to the multiplication by a scalar) to the global CLF $V_{\infty}$ outside a given compact set and equal to the local CLF $V_{0}$ on a given neighborhood of the origin (see below for a precise formulation of the problem under study). In Section II we exhibit a sufficient condition on $V_{0}$ and $V_{\infty}$ to piece together this pair of CLFs. Under this condition we design a new CLF $V$ solving our uniting problem. By focusing on an example we will check that this sufficient condition may not be satisfied for any pair of CLFs.

This problem is closely related to the possibility to piece together arbitrary local and global controllers. This uniting control problem has been introduced in [22] and further developed in [18]. More precisely, suppose we have a global controller $\phi_{\infty}$ which gives some nice properties for large

Vincent Andrieu and Christophe Prieur are with LAAS-CNRS, University of Toulouse, 7, avenue du Colonel Roche 31077 Toulouse, France vincent.andrieu@gmail.com, cprieur@laas.fr value of the state of a nonlinear system (e.g. a global attractivity of a compact set). Assume also that we have a local controller $\phi_{0}$ which ensures a nice behavior of the trajectories around the origin (e.g. a local exponential convergence). In [18] (see also [23]), the problem addressed was to synthesized a globally asymptotically stabilizing controller yielding the same qualitative behavior for large and small values of the state. This uniting control problem was solved by considering controllers with continuous and discrete dynamics (namely hybrid controller). As we will see in Section IV below, when the topological obstruction of [18] does not occur, solving the uniting CLF problem yields a simple solution to the uniting control problem without using discrete dynamics.

The problem of piecing together two CLFs seems to be challenging. Indeed, using the converse Lyapunov theory and the fact that it may be impossible to piece two arbitrary controllers when restricting to continuous stabilizing feedbacks, we understand that the uniting problem of two CLFs may not have any solution. As an illustration, a controlled system for which there exist two CLFs impossible to unite is introduced in Section IV. This obstruction motivates us to look for a sufficient condition guaranteeing the existence of a solution to the uniting CLF problem as done in Section II.

The paper is organized as follows. In Section II, we first state precisely the uniting CLF problem and then give a sufficient condition guaranteeing its solvability. In Section III, the linear case is investigated through a simple example. We introduce also a sufficient condition in terms of LMI. Section IV is devoted to the uniting control problem. In this section, we show how we can solve this problem once we have solved the uniting CLF problem. Finally in Section V, we present our conclusions.

The proof of some results has been removed due to space limitation.

Notation: $L_{f} V$ denotes the Lie derivative of a differentiable function $V$ with respect to the vector field $f$. Given a symmetric matrix $Q$, the notation $Q<0$ (resp. $Q \leq 0$ ) means that it is negative definite (resp. semi-definite).

\section{PROBLEM STATEMENT AND MAIN RESUlT}

\section{A. Problem formulation}

We consider a nonlinear system which is affine in its control and which is described by:

$$
\dot{x}=f(x)+g(x) u,
$$

where $x$ in $\mathbb{R}^{n}$ is the state, $u$ in $\mathbb{R}^{p}$ is the control input, and $f: \mathbb{R}^{n} \rightarrow \mathbb{R}^{n}$ and $g: \mathbb{R}^{n} \rightarrow \mathbb{R}^{p}$ are locally Lipschitz functions such that $f(0)=0$. 
We assume that two CLFs $V_{0}$ and $V_{\infty}$ satisfying Artstein condition on specific sets are given. More precisely we assume that the following assumption holds

Assumption 1: There exist a positive definite and continuously differentiable function $V_{0}: \mathbb{R}^{n} \rightarrow \mathbb{R}_{+}$, a positive semi-definite, proper and continuously differentiable function $V_{\infty}: \mathbb{R}^{n} \rightarrow \mathbb{R}_{+}$, and positive values $R_{0}$ and $r_{\infty}$ such that :

- Local CLF: for all $x$ in $\left\{x: 0<V_{0}(x) \leq R_{0}\right\}$, we have:

$$
L_{g} V_{0}(x)=0 \Rightarrow L_{f} V_{0}(x)<0 .
$$

- Global set-CLF: for all $x$ in $\left\{x: V_{\infty}(x) \geq r_{\infty}\right\}$, we have:

$$
L_{g} V_{\infty}(x)=0 \Rightarrow L_{f} V_{\infty}(x)<0 .
$$

- Covering assumption: we have the property:

$$
\left\{x: V_{\infty}(x)>r_{\infty}\right\} \cup\left\{x: V_{0}(x)<R_{0}\right\}=\mathbb{R}^{n} .
$$

Note that the Covering assumption is natural since we need that the two sets in which we have the controllability property (the two sets in which each CLF satisfies Artstein condition) overlap and cover the entire domain.

Let us define the problem we solve in this paper.

Uniting CLF problem: The uniting CLF problem is to find a proper, definite positive and continuously differentiable function $V: \mathbb{R}^{n} \rightarrow \mathbb{R}_{+}$such that:

- Global CLF: for all $x$ in $\mathbb{R}^{n} \backslash\{0\}$, we have:

$$
L_{g} V(x)=0 \quad \Rightarrow L_{f} V(x)<0 .
$$

- Local property: for all $x$ in $\left\{x: V_{\infty}(x) \leq r_{\infty}\right\}$, we have:

$$
V(x)=r_{\infty} V_{0}(x) .
$$

- Global property: for all $x$ in $\left\{x: V_{0}(x) \geq R_{0}\right\}$, we have:

$$
V(x)=R_{0} V_{\infty}(x) .
$$

One of the interest of this problematic is when the local CLF $V_{0}$ satisfies the small control property (see [21]) then in view of property (5) the same holds for the function $V$. In this case, we can use the so-called universal formulas (see [21], [14], [5]) to compute a controller which renders the origin a globally and asymptotically stable equilibrium.

Furthermore, as seen in Section IV resolving the uniting CLF problem provides a way to piece together arbitrary stabilizing controllers.

\section{B. A sufficient condition}

The main result of this paper establishes a sufficient condition guaranteeing the existence of a solution to the uniting CLF problem. To state it we need the following additional assumption.

Assumption 2: Given two positive values $r_{\infty}$ and $R_{0}$ and two functions $V_{0}: \mathbb{R}^{n} \rightarrow \mathbb{R}_{+}$and $V_{\infty}: \mathbb{R}^{n} \rightarrow \mathbb{R}_{+}$. Assume we have the following implication, for all $x$ in $\left\{x: V_{\infty}(x)>r_{\infty}, V_{0}(x)<R_{0}\right\}$,

$$
\begin{gathered}
\exists \lambda_{x}>0: L_{g} V_{0}(x)=-\lambda_{x} L_{g} V_{\infty}(x) \\
\Downarrow \\
L_{f} V_{0}(x)\left|L_{g} V_{\infty}(x)\right|+L_{f} V_{\infty}(x)\left|L_{g} V_{0}(x)\right|<0 .
\end{gathered}
$$

We are now in position to state our main result.

Theorem 1: Under Assumptions 1 and 2, there exists a solution of our uniting CLF problem. More precisely, under an appropriate choice of two continuously differentiable functions $\varphi_{0}: \mathbb{R}_{+} \rightarrow[0,1]$ and $\varphi_{\infty}: \mathbb{R}_{+} \rightarrow[0,1]$, the function $V: \mathbb{R}^{n} \rightarrow \mathbb{R}_{+}$defined by, for all $x$ in $\mathbb{R}^{n}$,

$$
\begin{aligned}
V(x)= & R_{0}\left[\varphi_{0}\left(V_{0}(x)\right)+\varphi_{\infty}\left(V_{\infty}(x)\right)\right] V_{\infty}(x) \\
& +r_{\infty}\left[1-\varphi_{0}\left(V_{0}(x)\right)-\varphi_{\infty}\left(V_{\infty}(x)\right)\right] V_{0}(x),
\end{aligned}
$$

is a proper, definite positive continuously differentiable function satisfying (4), (5), and (6).

Proof: The function $V_{\infty}$ being semi-definite and proper, the set $\left\{x: V_{\infty}(x) \leq r_{\infty}\right\}$ is a non empty compact subset and we can introduce $r_{0}$ the positive real number defined as:

$$
r_{0}=\max _{\left\{x: V_{\infty}(x) \leq r_{\infty}\right\}} V_{0}(x)
$$

If $\left\{x: V_{0}(x) \geq R_{0}\right\} \neq \emptyset$ we can also define $R_{\infty}$ as $^{1}$ :

$$
R_{\infty}=\min _{\left\{x: V_{0}(x) \geq R_{0}\right\}} V_{\infty}(x)
$$

In the case where $\left\{x: V_{0}(x) \geq R_{0}\right\}=\emptyset$ we pick any $R_{\infty}>r_{\infty}$.

Note that with the Covering assumption, we get that ${ }^{2}$ : $r_{0}<R_{0}$, and $r_{\infty}<R_{\infty}$. Inspired by the construction given in [1], the uniting CLF we propose is given in (8) where $\varphi_{0}$ and $\varphi_{\infty}$ are two continuously differentiable non decreasing functions satisfying:

$$
\begin{aligned}
& \varphi_{0}(s) \begin{cases}=0 & \forall s \leq r_{0}, \\
>0 & \forall r_{0}<s<R_{0}, \\
=\frac{1}{2} & \forall s \geq R_{0},\end{cases} \\
& \varphi_{\infty}(s) \begin{cases}=0 & \forall s \leq r_{\infty}, \\
>0 & \forall r_{\infty}<s<R_{\infty}, \\
=\frac{1}{2} & \forall s \geq R_{\infty} .\end{cases}
\end{aligned}
$$

The function $V_{0}$ being definite positive and the function $V_{\infty}$ being proper, it can be checked that $V$ is positive definite and proper. Moreover it satisfies the local and asymptotic properties given in Equations (5) and (6).

It remains to show that $V$ satisfies Artstein condition for all $x$ in $\mathbb{R}^{n} \backslash\{0\}$. Note that the functions $V_{0}$ and $V_{\infty}$ satisfying the implications (2) and (3), it yields that the function $V$ satisfies Artstein condition on the set $\{x$ : $\left.V_{0}(x) \geq R_{0}\right\} \cup\left\{x \neq 0: V_{\infty}(x) \leq r_{\infty}\right\}$. We need to show that Artstein condition is also satisfied on the set $\left\{x: V_{0}(x)<R_{0}, V_{\infty}(x)>r_{\infty}\right\}$. First of all, note that in this set, we have:

$$
R_{0} V_{\infty}(x)-r_{\infty} V_{0}(x)>0 .
$$

\footnotetext{
${ }^{1}$ Indeed, if we pick any element $x^{*}$ in $\left\{x: V_{0}(x) \geq R_{0}\right\}$, since the function $V_{\infty}$ is proper, we get that $\left\{x: V_{\infty}(x) \leq\right.$ $\left.V\left(x^{*}\right)\right\}$ is a compact set and we get $\min _{\left\{x: V_{0}(x) \geq R_{0}\right\}} V_{\infty}(x)=$ $\min _{\left\{x: V_{0}(x) \geq R_{0}, V_{\infty} \leq V\left(x^{*}\right)\right\}} V_{\infty}(x)$

${ }^{2}$ Otherwise there exists $x^{*}$ such that $V_{\infty}\left(x^{*}\right) \leq r_{\infty}$ and $V_{0}\left(x^{*}\right)>R_{0}$ which contradicts the Covering assumption.
} 
Furthermore, we have:

$$
\begin{aligned}
& L_{f} V(x)=A(x) L_{f} V_{0}(x)+B(x) L_{f} V_{\infty}(x), \\
& L_{g} V(x)=A(x) L_{g} V_{0}(x)+B(x) L_{g} V_{\infty}(x),
\end{aligned}
$$

where the continuous functions $A: \mathbb{R}^{n} \rightarrow \mathbb{R}_{+}$and $B$ : $\mathbb{R}^{n} \rightarrow \mathbb{R}_{+}$are defined as,

$$
\begin{aligned}
A(x)=\left[R_{0} V_{\infty}(x)-\right. & \left.r_{\infty} V_{0}(x)\right] \varphi_{0}^{\prime}\left(V_{0}(x)\right) \\
+r_{\infty} & {\left[1-\varphi_{0}\left(V_{0}(x)\right)-\varphi_{\infty}\left(V_{\infty}(x)\right)\right], } \\
B(x)=\left[R_{0} V_{\infty}(x)-\right. & \left.r_{\infty} V_{0}(x)\right] \varphi_{\infty}^{\prime}\left(V_{\infty}(x)\right) \\
& +R_{0}\left[\varphi_{0}\left(V_{0}(x)\right)+\varphi_{\infty}\left(V_{\infty}(x)\right)\right] .
\end{aligned}
$$

In the set $\left\{x: V_{0}(x)<R_{0}, V_{\infty}(x)>r_{\infty}\right\}$ we have $A(x)>0$ and $B(x)>0$. Suppose there exists $x^{*}$ in this set such that $L_{g} V\left(x^{*}\right)=0$. We have two cases:

- If $L_{g} V_{0}\left(x^{*}\right)=0$, then $L_{g} V_{\infty}\left(x^{*}\right)=0$, and since $V_{0}$ and $V_{\infty}$ satisfy the Artstein condition, this implies that $L_{f} V\left(x^{*}\right)<0$.

- If $L_{g} V_{0}\left(x^{*}\right) \neq 0$, this implies:

and

$$
L_{g} V_{0}\left(x^{*}\right)=-\frac{B\left(x^{*}\right)}{A\left(x^{*}\right)} L_{g} V_{\infty}\left(x^{*}\right),
$$

$$
A\left(x^{*}\right)=\frac{B\left(x^{*}\right)\left|L_{g} V_{\infty}\left(x^{*}\right)\right|}{\left|L_{g} V_{0}\left(x^{*}\right)\right|} .
$$

Consequently, with Assumption 2, we get:

$$
\begin{aligned}
L_{f} V\left(x^{*}\right) & =\frac{B\left(x^{*}\right)}{\left|L_{g} V_{0}\left(x^{*}\right)\right|}\left[L_{f} V_{0}\left(x^{*}\right)\left|L_{g} V_{\infty}\left(x^{*}\right)\right|\right. \\
& \left.+L_{f} V_{\infty}(x)\left|L_{g} V_{0}\left(x^{*}\right)\right|\right], \\
& <0 .
\end{aligned}
$$

This concludes the proof of Theorem 1.

Instead of considering CLFs, we may also consider weak CLFs. i.e. proper positive definite continuously differentiable functions such that for all $x$ in $\mathbb{R}^{n} \backslash\{0\}$ satisfying $L_{g} V(x)=$ 0 we have $L_{f} V(x) \leq 0$. In that framework it is possible to adapt Assumption 2 and Theorem 1.

\section{About Assumption 2}

Assumption 2 is a sufficient condition to solve the uniting CLF problem. Note that a way to relax this assumption is to restrict the sufficient condition in Theorem 1 to $\lambda_{x}=\frac{B(x)}{A(x)}$, where $A$ and $B$ are the continuous functions defined in the proof of Theorem 1.

Another formulation of this assumption can be given in terms of existence for each $x$ of a unique control $u_{x}$ rendering negative the time derivative of both $V_{0}$ and $V_{\infty}$. Indeed, we have the following proposition.

Proposition 1: Given two CLFs $V_{0}: \mathbb{R}^{n} \rightarrow \mathbb{R}_{+}$and $V_{\infty}:$ $\mathbb{R}^{n} \rightarrow \mathbb{R}_{+}$, and a state $x$ in $\mathbb{R}^{n} \backslash\{0\}$. The implication (7) is equivalent with the fact that there exists a control $u_{x}$ in $\mathbb{R}^{p}$ such that:

$$
\begin{aligned}
& L_{f} V_{0}(x)+L_{g} V_{0}(x) u_{x}<0, \\
& L_{f} V_{\infty}(x)+L_{g} V_{\infty}(x) u_{x}<0 .
\end{aligned}
$$

Proof: Proof of $(11) \Rightarrow(7)$ : Let $x^{*}$ in $\mathbb{R}^{n} \backslash\{0\}$ and $\lambda_{x^{*}}$ in $\mathbb{R}_{+}$be such that $L_{g} V_{0}\left(x^{*}\right)=-\lambda_{x} L_{g} V_{\infty}\left(x^{*}\right)$, and suppose there exists $u_{x^{*}}$ in $\mathbb{R}^{p}$ such that (11) is satisfied with $x=x^{*}$ and $u=u_{x^{*}}$. This implies:

$$
\begin{aligned}
L_{f} V_{0}\left(x^{*}\right)<-L_{g} V_{0}\left(x^{*}\right) u_{x^{*}} & =\lambda_{x^{*}} L_{g} V_{\infty}\left(x^{*}\right) u_{x^{*}}, \\
& <-\lambda_{x^{*}} L_{f} V_{\infty}\left(x^{*}\right) .
\end{aligned}
$$

Since $\lambda_{x^{*}}=\frac{\left|L_{g} V_{0}\left(x^{*}\right)\right|}{\left|L_{g} V_{\infty}\left(x^{*}\right)\right|}$ we get (7).

Proof of (7) $\Rightarrow$ (11): For the converse, suppose (7) is satisfied. We distinguish several cases. If $L_{g} V_{0}\left(x^{*}\right)=0$, since $x \neq 0$ and the function $V_{0}$ satisfies the Artstein condition, we have $L_{f} V_{0}\left(x^{*}\right)<0$. Consequently each control input $u_{x^{*}}$ such that $L_{f} V_{\infty}\left(x^{*}\right)+L_{g} V_{\infty}\left(x^{*}\right) u_{x^{*}}<0$ ensures that (11) is satisfied. We deal similarly with the case $L_{g} V_{\infty}\left(x^{*}\right)=0$. Hence, suppose that $L_{g} V_{0}\left(x^{*}\right) \neq 0$ and $L_{g} V_{\infty}\left(x^{*}\right) \neq 0$ and let $u_{x^{*}}$ be defined by:

$$
u_{x^{*}}=-k\left(\frac{L_{g} V_{0}\left(x^{*}\right)^{T}}{\left|L_{g} V_{0}\left(x^{*}\right)\right|}+\frac{L_{g} V_{\infty}\left(x^{*}\right)^{T}}{\left|L_{g} V_{\infty}\left(x^{*}\right)\right|}\right),
$$

where $k$ is a positive real number. Using the fact that

$$
\begin{aligned}
& L_{g} V_{0}\left(x^{*}\right) L_{g} V_{\infty}\left(x^{*}\right)^{T}= \\
& \quad\left|L_{g} V_{0}\left(x^{*}\right)\right|\left|L_{g} V_{\infty}\left(x^{*}\right)\right| \cos \left(L_{g} V_{0}\left(x^{*}\right), L_{g} V_{\infty}\left(x^{*}\right)\right),
\end{aligned}
$$

it yields:

$$
\begin{aligned}
L_{f} V_{0}\left(x^{*}\right) & +L_{g} V_{0}\left(x^{*}\right) u_{x^{*}}=L_{f} V_{0}\left(x^{*}\right) \\
& -k\left(1+\cos \left(L_{g} V_{0}\left(x^{*}\right), L_{g} V_{\infty}\left(x^{*}\right)\right)\left|L_{g} V_{0}\left(x^{*}\right)\right|,\right. \\
L_{f} V_{\infty}\left(x^{*}\right) & +L_{g} V_{\infty}\left(x^{*}\right) u_{x^{*}}=L_{f} V_{\infty}\left(x^{*}\right) \\
- & k\left(1+\cos \left(L_{g} V_{0}\left(x^{*}\right), L_{g} V_{\infty}\left(x^{*}\right)\right)\left|L_{g} V_{\infty}\left(x^{*}\right)\right| .\right.
\end{aligned}
$$

Suppose $\cos \left(L_{g} V_{0}\left(x^{*}\right), L_{g} V_{\infty}\left(x^{*}\right)\right)>-1$. In this case, we get easily the result taking $k$ sufficiently large. When $\cos \left(L_{g} V_{0}\left(x^{*}\right), L_{g} V_{\infty}\left(x^{*}\right)\right)=-1$ (i.e the upper condition in (7) is satisfied), by Assumption 2 we can select a real number $\mu_{x^{*}}$ such that:

$$
\frac{L_{f} V_{\infty}\left(x^{*}\right)}{\left|L_{g} V_{\infty}\left(x^{*}\right)\right|^{2}}<\mu_{x^{*}}<-\frac{L_{f} V_{0}\left(x^{*}\right)}{\left|L_{g} V_{0}\left(x^{*}\right)\right|\left|L_{g} V_{\infty}\left(x^{*}\right)\right|}
$$

If we consider the control input $u_{x^{*}}$ defined as:

$$
u_{x^{*}}=-\mu_{x^{*}} L_{g} V_{\infty}\left(x^{*}\right)^{T}
$$

we get using the second inequality of (12):

$$
\begin{aligned}
L_{f} V_{\infty}\left(x^{*}\right)+ & L_{g} V_{\infty}\left(x^{*}\right) u_{x^{*}} \\
& =L_{f} V_{\infty}\left(x^{*}\right)-\mu_{x^{*}}\left|L_{g} V_{\infty}\left(x^{*}\right)\right|^{2}, \\
& <0 .
\end{aligned}
$$

Employing the first inequality of (12) yields:

$$
\begin{aligned}
L_{f} V_{0}\left(x^{*}\right) & +L_{g} V_{0}\left(x^{*}\right) u_{x^{*}} \\
& =L_{f} V_{0}\left(x^{*}\right)-\mu_{x^{*}} L_{g} V_{0}\left(x^{*}\right) L_{g} V_{\infty}\left(x^{*}\right)^{T}, \\
& =L_{f} V_{0}\left(x^{*}\right)+\mu_{x^{*}}\left|L_{g} V_{0}\left(x^{*}\right)\right|\left|L_{g} V_{\infty}\left(x^{*}\right)\right|, \\
& <0 .
\end{aligned}
$$

This concludes the proof of Proposition 1. 
Assumption 2 is a sufficient condition which may not be satisfied given two CLFs. Indeed, consider the following nonlinear control system ${ }^{3}$ :

$$
\left\{\begin{array}{l}
\dot{x}_{1}=-x_{2}+\left(1+x_{1}\right) u \\
\dot{x}_{2}=x_{1}-x_{2} u
\end{array}\right.
$$

Let us consider the following continuously differentiable positive definite function $V_{\infty}\left(x_{1}, x_{2}\right)=x_{1}^{2}+x_{2}^{2}$ for all $\left(x_{1}, x_{2}\right) \in \mathbb{R}^{2}$. Note that for all $\left(x_{1}, x_{2}\right) \in \mathbb{R}^{2}$,

$$
L_{g} V_{\infty}\left(x_{1}, x_{2}\right)=2 x_{1}+2 x_{1}^{2}-2 x_{2}^{2}
$$

and

$$
L_{f} V_{\infty}\left(x_{1}, x_{2}\right)=0 .
$$

Thus $V_{\infty}$ is a global (weak) CLF.

Consider the local homogenous approximation with weights $r_{x_{1}}=1, r_{x_{2}}=2$ and $r_{u}=0$ which is

$$
\dot{x}_{1}=u \quad, \quad \dot{x}_{2}=x_{1} .
$$

We define $V_{0}$ as being the minimal time function with the constraint $|u| \leq 1$ to reach the origin which may be computed with Hamilton-Jacobi theory (see [12, Section 5], e.g.):

$$
V_{0}=\left\{\begin{array}{l}
2 \sqrt{x_{1}^{2} / 2+x_{2}}+x_{1} \text { if } x_{2}+\frac{x_{1}^{2}}{2} \operatorname{sign}\left(x_{1}\right) \geq 0 \\
2 \sqrt{x_{1}^{2} / 2-x_{2}}-x_{1} \text { if } x_{2}+\frac{x_{1}^{2}}{2} \operatorname{sign}\left(x_{1}\right)<0
\end{array}\right.
$$

The function $V_{0}$ satisfies (weak) Artstein condition locally around the origin and is continuously differentiable for all $\left(x_{1}, x_{2}\right)$ such that $x_{2}+\frac{x_{1}^{2}}{2} \operatorname{sign}\left(x_{1}\right) \neq 0$. Moreover, for all $\left(x_{1}, x_{2}\right)$ such that $x_{2}-\frac{x_{1}^{2}}{2}<0$ and $x_{1}<0$ we have:

$$
V_{0}\left(x_{1}, x_{2}\right)=2 \sqrt{-x_{2}+\frac{x_{1}^{2}}{2}}-x_{1},
$$

and hence,

$$
L_{f} V_{0}\left(x_{1}, x_{2}\right)=x_{2}+\frac{-x_{2} x_{1}-x_{1}}{\sqrt{-x_{2}+\frac{x_{1}^{2}}{2}}},
$$

and

$$
L_{g} V_{0}\left(x_{1}, x_{2}\right)=-1-x_{1}+\frac{-\left(1+x_{1}\right) x_{1}+x_{2}}{\sqrt{-x_{2}+\frac{x_{1}^{2}}{2}}} .
$$

Moreover, combining (14), (15), (16), and (17), and picking $x_{1}=-\sqrt{t}$ and $x_{2}=\frac{t}{4}$, with $t>0$ close to the zero yields

$$
\begin{aligned}
& \left.L_{g} V_{0}\left(x_{1}, x_{2}\right)\right)=1+\frac{1}{4} \sqrt{t} \sim_{t \rightarrow 0} 1>0, \\
& L_{g} V_{\infty}\left(x_{1}, x_{2}\right)=-2 \sqrt{t}+2 t-\frac{1}{8} t^{2} \sim_{t \rightarrow 0}-2 \sqrt{t}<0 \\
& L_{f} V_{0}\left(x_{1}, x_{2}\right)=\frac{3}{4} t+2 \sim_{t \rightarrow 0} 2>0 \\
& L_{f} V_{\infty}\left(x_{1}, x_{2}\right)=0 \sim_{t \rightarrow 0} 0 .
\end{aligned}
$$

${ }^{3}$ System (13) is derived from the Gauss equation. :

$$
\begin{aligned}
\dot{L} & =\varpi(p, \varepsilon)-\Im(\eta) W, \\
\dot{p} & =2 p S \\
\dot{\varepsilon} & =-j \varphi(p, \varepsilon) \varepsilon+(\varepsilon+2+\Re(\varepsilon)) S, \\
\dot{\eta} & =-j[\varpi(p, \varepsilon)-\Im(\eta) W] \eta+\frac{1}{2}\left(1+|\eta|^{2}\right) W,
\end{aligned}
$$

by approximating $\varpi(p, \varepsilon)$ by 1 , and by letting $x_{1}=\Re(\varepsilon), x_{2}=-\Im(\varepsilon)$, and $u=\frac{1}{2} S$, and where $\Re(\varepsilon)$ and $\Im(\varepsilon)$ denote respectively the real and the imaginary part of $\varepsilon: \varepsilon=\Re(\varepsilon)+j \Im(\varepsilon)$.
Consequently for $t$ sufficiently small the condition (7) is not satisfied. And the functions $V_{0}$ and $V_{\infty}$ do not satisfy Assumption 2 of Theorem 1 for $R_{0}$ small enough.

\section{UNITING TWO CLFS IN THE LINEAR CASE}

In this Section, the system (1) is supposed to be linear, i.e. we suppose there exist two matrices $F$ in $\mathbb{R}^{n \times n}$ and $G$ in $\mathbb{R}^{n \times p}$ such that the system (1) can be rewritten as:

$$
\dot{x}=F x+G u \text {. }
$$

In the linear framework, the CLFs are defined as $V_{0}(x)=$ $x^{T} P_{0} x$ and $V_{\infty}(x)=x^{T} P_{\infty} x$ where $P_{0}$ and $P_{\infty}$ are symmetric definite positive matrices in $\mathbb{R}^{n \times n}$ such that:

$$
\begin{aligned}
& x^{T} P_{0} G=0 \quad \Rightarrow \quad x^{T}\left(F^{T} P_{0}+P_{0} F\right) x<0 \\
& x^{T} P_{\infty} G=0 \quad \Rightarrow \quad x^{T}\left(F^{T} P_{\infty}+P_{\infty} F\right) x<0 .
\end{aligned}
$$

Despite that for linear systems all quadratic CLFs satisfies global and local properties, for robustness issue or qualitative behavior, it may be of interest to unit a pair of CLFs (see Section IV for an illustration).

\section{A. Case of a chain of integrators of order 2}

To illustrate Theorem 1, we consider a simple case where the state of the linear system (18) is in $\mathbb{R}^{2}$ and where we pick:

$$
F=\left(\begin{array}{ll}
0 & 1 \\
0 & 0
\end{array}\right) \quad, \quad G=\left(\begin{array}{l}
0 \\
1
\end{array}\right)
$$

The matrices $P_{0}$ and $P_{\infty}$ are any positive definite matrices in $\mathbb{R}^{2 \times 2}$ defined as: ${ }^{4}$

$$
P_{0}=\left(\begin{array}{cc}
a_{0} & b_{0} \\
\star & c_{0}
\end{array}\right), \quad P_{\infty}=\left(\begin{array}{cc}
a_{\infty} & b_{\infty} \\
\star & c_{\infty}
\end{array}\right) .
$$

These matrices are definite positive if and only if

$$
\begin{array}{lll}
a_{0}>0, & c_{0}>0 \quad, & a_{0} c_{0}-b_{0}^{2}>0, \\
a_{\infty}>0 & c_{\infty}>0, & a_{\infty} c_{\infty}-b_{\infty}^{2}>0 .
\end{array}
$$

Note also that (19) is satisfied if and only if

$$
b_{0}>0, \quad b_{\infty}>0 .
$$

The interest of this system is that the sufficient condition introduced in Theorem 1 can always be satisfied provided the two real numbers $R_{0}$ and $r_{\infty}$ are selected in an appropriate way. Indeed, for this particular system, we have the following result.

Proposition 2: For all $P_{0}$ and $P_{\infty}$ satisfying (21) and (22), and for all $R_{0}, r_{\infty}$ satisfying

$$
r_{\infty} P_{0}-R_{0} P_{\infty} \leq 0
$$

we can construct a solution to the uniting CLF problem, i.e. a CLF for the system (18) with $F$ and $G$ defined in (20) which satisfies:

$$
V(x)= \begin{cases}r_{\infty} x^{T} P_{0} x \quad & \forall x: x^{T} P_{\infty} x \leq r_{\infty} \\ R_{0} x^{T} P_{\infty} x & , \quad \forall x: x^{T} P_{0} x \geq R_{0}\end{cases}
$$

\footnotetext{
${ }^{4}$ For any symmetric matrix, we will denote the symmetric terms by $\star$.
} 


\section{B. System of higher dimension}

For system of higher dimension, Assumption 2 might be difficult to check. Nevertheless, given two symmetric positive definite matrices $P_{0}$ and $P_{\infty}$ defining two CLFs, we can express a stronger sufficient condition in terms of LMI:

Proposition 3: If there exists a matrix $K$ in $\mathbb{R}^{n \times p}$ such that the following LMIs are satisfied

$$
\left\{\begin{array}{l}
(F+G K)^{T} P_{0}+P_{0}(F+G K)<0 \\
(F+G K)^{T} P_{\infty}+P_{\infty}(F+G K)<0
\end{array}\right.
$$

then for all positive real numbers $R_{0}$ and $r_{\infty}$ satisfying (23), there exists a $C^{1}$, positive definite and proper function $V$ : $\mathbb{R}^{n} \rightarrow \mathbb{R}_{+}$which is a global CLF for the system (18) and such that (24) is satisfied.

Note that Assumption 2 may not be satisfied even for linear systems. Consider e.g. the control system (18) with:

$$
F=\left(\begin{array}{ccc}
1.1 & -0.76 & -1.1 \\
1.6 & 0.44 & 0.20 \\
1.4 & 0.91 & 0.76
\end{array}\right), \quad B=\left(\begin{array}{c}
-1.3 \\
-0.95 \\
0.78
\end{array}\right)
$$

The following symmetric positive definite matrices:

$$
P_{0}=\left(\begin{array}{ccc}
73 & -70 & 30 \\
\star & 121 & 10 \\
\star & \star & 48
\end{array}\right), \quad P_{\infty}=\left(\begin{array}{ccc}
3 & 1 & 1 \\
\star & 5 & 3 \\
\star & \star & 2
\end{array}\right),
$$

are such that $x \mapsto x^{T} P_{0} x$ and $x \mapsto x^{T} P_{\infty} x$ are two $\mathrm{CLFs}^{5}$. We may check that for $x^{*}=(-1.5,1,-0.5)^{T}$, $L_{g} V_{\infty}\left(x^{*}\right) L_{g} V_{0}\left(x^{*}\right)<0$ and that:

$$
L_{f} V_{\infty}\left(x^{*}\right)\left|L_{g} V_{0}\left(x^{*}\right)\right|+L_{f} V_{0}\left(x^{*}\right)\left|L_{g} V_{\infty}\left(x^{*}\right)\right|>0 .
$$

Therefore Assumption 2 is not satisfied.

For a chain of integrators of order 3 , we do not know if Assumption 2 holds for each couple of quadratic CLFs $\left(V_{0}, V_{\infty}\right)$. However we can show that it is satisfied for a particular selection of quadratic functions $V_{0}$ and $V_{\infty}$. Indeed, we may check that picking

$P_{0}=\left(\begin{array}{ccc}4.72 & 8.06 & 1.00 \\ \star & 37.3 & 6.72 \\ \star & \star & 11.1\end{array}\right), P_{\infty}=\left(\begin{array}{ccc}2.02 & 2.53 & 1.84 \\ \star & 4.39 & 2.89 \\ \star & \star & 3.33\end{array}\right)$

the functions $x \mapsto x^{T} P_{0} x$ and $x \mapsto x^{T} P_{\infty} x$ are two CLFs for (18) such that the LMIs (25) are satisfied with the vector $K=\left(\begin{array}{lll}-0.6535 & -2.0728 & -2.0986\end{array}\right)$. Therefore Assumption 2 holds and we succeed to solve the uniting problem for these CLFs by applying Proposition 3. Analogous examples can be found for systems of order 4 .

\section{ApPlicAtion to THE DESIGN OF A UNITING CONTROLLER}

\section{A. A general construction}

Our main result is instrumental in the design of a global asymptotic stabilizing feedbacks law. To the best of our knowledge, the use of several CLFs to design a controller is uncommon in control theory (although some results exist for

\footnotetext{
${ }^{5}$ Both are computed as solution of a Riccati equation.
}

the synthesis problem of hybrid controllers, see in particular [7]).

Here the result provided by Theorem 1 is a simple way to solve the uniting controller design problem. This problem has been introduced in [22] and further developed in [18]. In our context, we get the following proposition.

Proposition 4: Consider two functions $V_{0}: \mathbb{R}^{n} \rightarrow \mathbb{R}_{+}$ and $V_{\infty}: \mathbb{R}^{n} \rightarrow \mathbb{R}_{+}$and two positive real numbers $R_{0}$ and $r_{\infty}$ satisfying Assumptions 1 and 2 with the extra assumption that $V_{0}$ is proper. For any continuous functions $\phi_{0}: \mathbb{R}^{n} \rightarrow$ $\mathbb{R}^{p}$ satisfying for all $x$ in $\left\{x: 0<V_{0}(x) \leq R_{0}\right\}$

$$
L_{f} V_{0}(x)+L_{g} V_{0}(x) \phi_{0}(x)<0,
$$

and any function $\phi_{\infty}: \mathbb{R}^{n} \rightarrow \mathbb{R}^{p}$ satisfying for all $x$ in $\left\{x: V_{\infty}(x) \geq r_{\infty}\right\}$

$$
L_{f} V_{\infty}(x)+L_{g} V_{\infty}(x) \phi_{\infty}(x)<0,
$$

we can find another continuous function $\phi: \mathbb{R}^{n} \rightarrow \mathbb{R}^{p}$ which is a uniting controller, i.e. such that

1) $\phi(x)=\phi_{0}(x)$ for all $x$ such that $V_{\infty}(x) \leq r_{\infty}$,

2) $\phi(x)=\phi_{\infty}(x)$ for all $x$ such that $V_{0}(x) \geq R_{0}$,

3) the origin of the system $\dot{x}=f(x)+g(x) \phi(x)$ is a globally and asymptotically stable equilibrium.

Proof: To prove this result we construct the Control Lyapunov Function $V: \mathbb{R}^{n} \rightarrow \mathbb{R}_{+}$obtained from Theorem 1 . The control law is defined as:

$$
\phi(x)=\mathcal{H}(x)-k c(x) L_{g} V(x)
$$

with $\mathcal{H}(x)=\gamma(x) \phi_{0}(x)+[1-\gamma(x)] \phi_{\infty}(x)$ where $\gamma$ is any continuous function

$$
\gamma(x)= \begin{cases}1 & \text { if } V_{\infty}(x) \leq r_{\infty} \\ 0 & \text { if } V_{0}(x) \geq R_{0}\end{cases}
$$

the function $c$ is any continuous function such that

$$
c(x) \begin{cases}=0 & \text { if } V_{0}(x) \geq R_{0} \text { or } V_{\infty}(x) \leq r_{\infty}, \\ >0 & \text { if } V_{0}(x)<R_{0} \text { and } V_{\infty}(x)>r_{\infty},\end{cases}
$$

and $k$ is a positive real number defined later. Note that the function $\phi$ satisfies point 1) and 2) of Proposition 4. It remains to show point 3). Taking the function $V$ as a candidate Lyapunov function, we get:

$$
\begin{aligned}
\dot{V}(x)=\frac{\partial V}{\partial x}(x) f(x)+\frac{\partial V}{\partial x}(x) & g(x) \mathcal{H}(x) \\
& -k c(x)\left|\frac{\partial V}{\partial x}(x) g(x)\right|^{2} .
\end{aligned}
$$

With the local and global properties of the function $V$ (see Theorem 1), we have that for all $x$ in $\left\{x \neq 0: V_{\infty}(x) \leq\right.$ $r_{\infty}$ or $\left.V_{0}(x) \geq R_{0}\right\}$ :

$$
\dot{V}(x)<0 .
$$

We will show that if $k$ is selected sufficiently large then this control law ensures the negativeness of $\dot{V}$ on the whole domain. Indeed, suppose the assertion is wrong and suppose for each $k$ in $\mathbb{N}$, there exists $x_{k}$ in $\mathbb{R}^{n} \backslash\{0\}$ such that

$$
\dot{V}\left(x_{k}\right) \geq 0 \quad, \quad \forall k \in \mathbb{N} \text {. }
$$


With (28), $\left(x_{k}\right)_{k \in \mathbb{N}}$ is a sequence living in a compact set $\left\{x: V_{\infty}(x) \geq r_{\infty}\right\} \cap\left\{x: V_{0}(x) \leq R_{0}\right\}$. This subset being compact there exists a converging subsequence $\left(x_{k_{\ell}}\right)_{\ell \in \mathbb{N}}$ which converges to a point denoted $x^{*}$. The function $x \mapsto$ $\dot{V}(x)$ being continuous, we get $\dot{V}\left(x^{*}\right) \geq 0$. This implies that $x^{*}$ is in $\left\{x: V_{\infty}(x)>r_{\infty}\right\} \cap\left\{x: V_{0}(x)<R_{0}\right\}$, and that $c\left(x^{*}\right)>0$. Consequently, this implies that, $\frac{\partial V}{\partial x}\left(x^{*}\right) g\left(x^{*}\right)=$ 0 . The function $V$ being a CLF, this contradicts Assertion (29) and establishes that (28) is satisfied for all $x \neq 0$. Hence, Point 3 ) is also satisfied.

This proposition shows that once we have solved the uniting problem for two CLFs we get an answer to the uniting two controllers problem.

Note also that in [18] a topological necessary condition for the existence of a solution to the uniting control problem is exhibited. More precisely the system

$$
\dot{x}=-y^{2} u, \quad \dot{y}=u,
$$

is studied in [18]. The pair of controllers $\phi_{0}(x, y)=-y+x$ and $\phi_{\infty}(x, y)=-y-x$, ensuring that the origin of each closed loop system is a global and asymptotically stable equilibrium, are also introduced. It is shown in [18] that there does not exist any (static continuous) controller which is equal to the local controller in a neighborhood of the origin, and equal to the global controller outside of a compact set.

Employing converse Lyapunov theorems, we can associate two Lyapunov functions $V_{0}$ and $V_{\infty}{ }^{6}$ to each of these closed loop systems. These two Lyapunov functions define two CLFs which cannot be united (otherwise with Proposition 4 , we would be able to design a uniting controller).

\section{B. Illustration for the chain of integrators of order 2}

In view of Propositions 2 and 4, this implies that for the chain of integrators of order 2, each pair of linear stabilizing control laws can be united.

Proposition 5: Let $K_{0}$ and $K_{\infty}$ in $\mathbb{R}^{1 \times 2}$ be such that the origin of the systems:

$$
\dot{x}=\left(F+G K_{0}\right) x, \quad \dot{x}=\left(F+G K_{\infty}\right) x .
$$

where $F$ and $G$ are given in (20), is globally and asymptotically stable. Then for all positive real numbers $R_{0}$ and $r_{\infty}$ satisfying (23), there exists a continuous function $\phi: \mathbb{R}^{n} \rightarrow$ $\mathbb{R}^{p}$ such that the origin of the system $\dot{x}=F x+G \phi(x)$ is globally and asymptotically stable and such that:

$$
\phi(x)= \begin{cases}K_{0} x, & V_{\infty}(x) \leq r_{\infty} \\ K_{\infty} x, & V_{0}(x) \geq R_{0} . \\ \text { V. CONCLUSION }\end{cases}
$$

In this paper we have introduced a new problem which is to piece together two Control Lyapunov Functions. This problem provides a solution to the problem of uniting two controllers. The sufficient conditions given are always satisfied in the case of a simple chain of integrators.

\footnotetext{
${ }^{6}$ Note that the Lyapunov function introduced in [18] is the same for each controllers but this one cannot be used directly in Proposition 4 since this one is a weak Lyapunov function. Note however that strict CLFs can be obtained following the algorithm given in [15].
}

\section{ACKNOWLEDGEMENT}

The authors are deeply grateful to Laurent Praly and Alessandro Astolfi for helpful suggestions.

\section{REFERENCES}

[1] V. Andrieu, L. Praly, and A. Astolfi. Homogeneous Approximation, recursive observer design and output feedback. SIAM Journal of Control and Optimization, 47(4):1814-1850, 2008.

[2] Z. Arststein. Stabilization with relaxed controls. Nonlinear analysis, 7(11):1163-1173, 1983.

[3] F.H. Clarke, Y.S. Ledyaev, E.D. Sontag, and A.I. Subbotin. Asymptotic controllability implies feedback stabilization. IEEE Transactions on Automatic Control, 42(10):1394-1407, 1997.

[4] F.H. Clarke, Y.S. Ledyaev, and R.J. Stern. Asymptotic stability and smooth Lyapunov functions. Journal of Differential Equations, 149(1):69-114, 1998

[5] R. A. Freeman and P.V. Kokotovic. Inverse optimality in robust stabilization. SIAM Journal of Control Optimization, 34(4):13651391, 1996.

[6] R.A. Freeman and P.V. Kokotovic. Robust Nonlinear Control Design: State-Space and Lyapunov Techniques. Birkhauser, 1996.

[7] R. Goebel, C. Prieur, and A.R. Teel. Smooth patchy control Lyapunov functions. In 45th IEEE Conference on Decision and Control, pages 3271-3276, 2006.

[8] A. Isidori. Nonlinear control systems: an introduction. Springer-Verlag New York, Inc. New York, NY, USA, 1989.

[9] M. Jankovic, R. Sepulchre, and PV Kokotovic. Constructive Lyapunov stabilization of nonlinear cascade systems. IEEE Transactions on Automatic Control, 41(12):1723-1735, 1996.

[10] C.M. Krstic and H. Deng. Stabilization of Nonlinear Uncertain Systems. Springer-Verlag, London, 1998.

[11] M. Krstic, P.V. Kokotovic, and I. Kanellakopoulos. Nonlinear and Adaptive Control Design. John Wiley \& Sons, Inc. New York, NY, USA, 1995.

[12] J.B. Lasserre, D. Henrion, C. Prieur, and E. Trélat. Nonlinear optimal control via occupation measures and LMI-relaxations. SIAM Journal on Control and Optimization, 47(4):1643-1666, 2008.

[13] Y.S. Ledyaev and E.D. Sontag. A Lyapunov characterization of robust stabilization. Nonlinear Analysis, 37(7):813-840, 1999.

[14] Y. Lin and E.D. Sontag. A universal formula for stabilization with bounded controls. Systems \& Control Letters, 16(5):393-397, 1991.

[15] F. Mazenc and D. Nesic. Strong Lyapunov functions for systems satisfying the conditions of LaSalle. IEEE Transactions on Automatic Control, 49(6):1026-1030, 2004.

[16] F. Mazenc and L. Praly. Adding integrations, saturated controls, and stabilization forfeedforward systems. IEEE Transactions on Automatic Control, 41(11):1559-1578, 1996.

[17] L. Praly, B. Andréa-Novel, and J.-M. Coron. Lyapunov design of stabilizing controllers for cascaded systems. IEEE Transactions on Automatic Control, 36(10):1177-1181, 1991.

[18] C. Prieur. Uniting Local and Global Controllers with Robustness to Vanishing Noise. Mathematics of Control, Signals, and Systems (MCSS), 14(2):143-172, 2001.

[19] R. Sepulchre, M. Janković, and P.V. Kokotović. Constructive nonlinear control. Communications and Control Engineering Series. SpringerVerlag, 1997.

[20] E.D. Sontag. A Lyapunov-Like Characterization of Asymptotic Controllability. SIAM Journal on Control and Optimization, 21:462471, 1983.

[21] E.D. Sontag. A "universal" construction of Artstein's theorem on nonlinear stabilization. Systems \& control letters, 13(2):117-123, 1989.

[22] A.R. Teel, O.E. Kaiser, and R.M. Murray. Uniting local and global controllers for the Caltech ducted fan. In 16th American Control Conference, pages 539 - 1543, Albuquerque NM, 1997.

[23] A.R. Teel and N. Kapoor. Uniting local and global controllers. In European Control Conference, volume 172, 1997. 